\title{
An Overview of Maternal Anxiety During Pregnancy and the Post-Partum Period
}

\author{
Sarah Araji' , Ashley Griffin², Laura Dixon ${ }^{3,4}$, Shauna-Kay Spencer' ${ }^{1}$ Charlotte Peavie', Kedra Wallace ${ }^{1,5 *}$ \\ 'Department of Obstetrics \& Gynecology, University of Mississippi Medical Center, Jackson, MS, 39216, USA \\ ${ }^{2}$ Program in Neuroscience, University of Mississippi Medical Center, Jackson, MS, 39216 USA \\ ${ }^{3}$ Department of Psychology, University of Mississippi, Oxford, MS 38677 USA; \\ ${ }^{4}$ Department of Dermatology, University of Mississippi Medical Center, Jackson, MS, 39216 USA \\ ${ }^{5}$ Department of Neurobiology \& Anatomical Sciences, University of Mississippi Medical Center, Jackson, MS, 39216 USA
}

Article Info

\section{Article Notes}

Received: October 8, 2020

Accepted: November 30, 2020

\section{*Correspondence:}

Dr. Kedra Wallace, PhD, Associate Professor, University of Mississippi Medical Center, Jackson, Mississippi, United States; Telephone No: (601) 984-5396; Fax No: (601) 978-9974; Email: Kwallace2@umc.edu.

(C) 2020 Wallace $\mathrm{K}$. This article is distributed under the terms of the Creative Commons Attribution 4.0 International License.

\section{Keywords:}

Anxiety

Perinatal period

Post-partum anxiety

Pregnancy

Prenatal stress

\section{Abstract}

Anxiety during pregnancy is associated with adverse outcomes in mothers and infants. Unfortunately, as anxiety is often synonymously mentioned with depression, the studies focusing solely on anxiety during pregnancy are not as robust as those in the field of depression are. In this work, we provide an overview of what is currently known about general anxiety during pregnancy, pregnancy-related anxiety and the potential impacts anxiety could have on post-partum care. An overview of potential risk factors, post-partum maternal outcomes, infant outcome along with pharmacological and nonpharmacological treatments are covered with a specific focus on high-risk pregnancies. Although anxiety during pregnancy is normative; anxiety can become problematic and negatively impact upon behavior, resulting in potential harm to the mother, as well as her developing fetus or child (ren) at home. The clinical diagnosis for anxiety and conditions associated with anxiety often require lengths of time that are not applicable for the pregnant patient, which has led to diagnosis and terms such as pregnancy-related anxiety. Importantly, increasing awareness about the increased potential risk to mothers who may be affected by anxiety during pregnancy or the post-partum period has the potential to improve maternal mental health screening and access to care.

\section{Introduction}

Anxiety and depression during pregnancy are significant complications that have been reported to affect between $20-40 \%$ of pregnant women ${ }^{1,2}$. Women are disproportionately affected by mood and anxiety disorders, especially in the childbearing age, and mental health issues often worsen or emerge during pregnancy. With more than 500,000 women in the United States either having a preexisting psychiatric illness or being diagnosed with one during pregnancy ${ }^{3}$, this is an area of growing concern. The general causes of worries during pregnancy can revolve around: fetal wellbeing, maternal illnesses, social and financial support and mortality ${ }^{4}$. If these worries persist for a prolonged period, they can lead to functional impairment across multiple areas of the mother's life ${ }^{5}$. Despite these facts, the majority of studies and reviews to date focus on depression or anxiety in the presence of depression. There are very few studies that focus on anxiety alone in pregnancy which led us to conduct this overview. As such the purpose of the current overview of the literature is to describe common expressions of anxiety disorders occurring during pregnancy, identify risk factors associated with anxiety disorders, evaluate some of the potential outcomes of pre- and post-partum anxiety disorders and provide 
an overview of current recommendations for treatment. A comprehensive search strategy on PubMed/Medline database was performed to identify studies written in English and focused on anxiety during pregnancy. Publications published between 2010-2020 were primarily focused on, however original work from older publications were also included as deemed appropriate. The search strategy included the following terms and or phrases: postpartum anxiety, pregnancy anxiety, prenatal stress, and antepartum anxiety. Additionally, work specific to cognitive behavioral therapy (CBT) and home visit programs (HVPs) or manuscripts identified from the chosen articles were also utilized.

\section{Anxiety and Pregnancy}

Anxiety and depression disorders are highly co-morbid, with $30-58 \%$ of pregnant patients meeting the criteria for both conditions ${ }^{6}$. As such, studies examining the prevalence of solely anxiety disorders in pregnant women, without depression disorders, are limited. Generalized anxiety disorder (GAD) is diagnosed when the following characteristics are present for at least 6 months: excessive worry, unrealistic view of problems, irritability, and the manifestation of physical symptoms (e.g., headaches, nausea, tension $)^{7}$. The prevalence of GAD in the nonpregnant population ranges from $5.1-11.9 \%$, with females being twice as likely as males to be affected ${ }^{8,9}$.

Pregnancy represents a time point in which anxiety may either be exacerbated or have new onset in women's lives; studies have estimated that at least $10.8 \%$ of pregnant women are affected by GAD ${ }^{10,11}$. In fact, a study by Phillips et al. reported that $14.3 \%$ of mothers stated onset of anxiety at childbirth, $20 \%$ of women recognized the onset of new anxiety during pregnancy and $51.4 \%$ immediately after childbirth ${ }^{10}$. To further complicate matters, the majority of pregnant women with anxiety were found to have worries not associated with GAD; leading to pregnancy-related anxiety (PrA) which is suggested to operate as a separate disorder ${ }^{12}$. PrA has been suggested to have origins of the worry associated specifically with maternal and/or infant outcomes, as opposed to GAD which is generalized anxiety ${ }^{13}$. A study by Huzinik et al. explored PrA in the following three different sub-areas: fear of giving birth, fear of having an impaired child, and fear of maternal physical appearance ${ }^{12}$. In this study, many of the worries of pregnancy decreased towards the third trimester whereas women who were continuously anxious during their pregnancy had increased anxiety in the third trimester ${ }^{12}$. This increase in anxiety is believed to be one of the primary differentiations between GAD and PrA. A concept analysis of PrA by Bayrampour et al., further expanded the definition of PrA to include experiences with the health care system, pregnancy-related social and financial issues, and parenting ${ }^{13}$. This analysis continued to categorize PrA into three primary attributes (i.e., affective responses, cognitions and somatic symptoms) with three behavioral consequences (i.e., negative attitudes, excessive reassurance-seeking behavior, and avoidance behavior). In a recent study, women with a history of GAD or social anxiety were found to be at an increased risk of developing $\operatorname{PrA}^{14}$.

Obsessive-compulsive disorder (OCD) is described by a chronic pattern of unwanted, intrusive thoughts and repetitive behaviors that increases anxiety, affecting productivity, which can develop or worsen during pregnancy ${ }^{15}$. Women who have OCD prior to pregnancy report an escalation in compulsions during pregnancy that progresses and even worsens into the post-partum period $^{16}$. In a recent review, Viswasam et al. reported that up to $39 \%$ of women with new onset OCD during pregnancy also had worsening compulsivity as the pregnancy progressed, which increased in the post-partum period $^{17}$. Anxiety during pregnancy has been described as a normal experience in the anticipation of childbirth, and when anxiety is present at low levels, it may be beneficial in preparing one for parenthood ${ }^{18}$. However, regardless of the type of anxiety disorder a pregnant woman experiences, anxiety has the potential to become distressing and interfere with one's life ${ }^{5}$. At more severe levels, anxiety is uncontrollable and results in negative behavioral outcomes, indicating the need for a more thorough psychological evaluation to determine the presence of a potential anxiety disorder diagnosis.

\section{Risk factors associated with anxiety during pregnancy}

High risk pregnancy and labor are two major physiological risk factors associated with anxiety during pregnancy and will be discussed in detail. However, it is important to realize that other risk factors for anxiety disorders exists and can include environmental, physical, biological, social, and psychological determinants that may predispose an individual to develop an anxiety disorder in the antepartum, intrapartum or post-partum period $(s)^{14,19}$. While all of these factors may have an additive effect on the anxiety state of an individual it is important to also realize that each factor can also individually lead to increased anxiety. Environmental factors can include daily life stressors or hassles related to daily responsibilities at home or at work ${ }^{20,21}$. Additionally, the amount of social support received can also contribute to anxiety disorders as the availability of intimate partner support has been reported to serve as a predictor of anxiety in pregnancy ${ }^{22}$. Other factors such as socioeconomic status (income, employment and education) is also predictive of the likelihood of either increasing anxiety and/or developing anxiety disorders during pregnancy ${ }^{23,24}$. 


\section{High-risk pregnancy and anxiety disorders}

Between 15-20\% of pregnancies are high-risk, wherein the pregnancy is complicated by one or more serious condition(s) that affect maternal and/or fetal outcome $^{25}$. Unfortunately, this status may exacerbate the stress associated with normal pregnancies, thereby further increasing anxiety and risk in a cyclical manner ${ }^{26}$. Fairbrother et al. reported that women with high-risk pregnancies were found to have a $5.2 \mathrm{x}$ greater incidence of anxiety compared to women with low-risk pregnancies ${ }^{27}$. There are multiple factors that will label pregnancy as high risk. We will discuss a few of them and their association with anxiety in pregnancy.

High-risk pregnancies require an increased level of care, more frequent physician visits, and closer follow-up, which imposes additional financial and time constraints for the patient. Although the chronicity of the associated high-risk illness may increase adaptability, women may experience additional fears related to the illness affecting pregnancy outcomes, such as personal and fetal/infant health ${ }^{13,24}$. Additionally, Martini et al, found that women who were older than 35 at childbirth or who had histories of miscarriages were more prone to anxiety ${ }^{24}$. On the other hand, newly diagnosed illnesses may increase the risk for anxiety due to new life adjustments and health regiments. Pre-gestational diabetes ${ }^{28}$ and preeclampsia (new onset of hypertension accompanied by proteinuria and/or severe headaches ${ }^{29}$ ) are among the more common chronic illnesses seen in pregnancies. Both conditions are associated with prolonged hospitalization, and increased maternal and fetal morbidity and mortality ${ }^{30}$. Patients affected by these conditions report higher levels of anxiety related to the effect of their illnesses on their fetus and pregnancy progress. Not to undermine the importance of obstetric complications both with and without high-risk pregnancies on anxiety, a recent review of 26 studies found that $20-100 \%$ of women with an obstetric complication (unplanned surgical, social and/or obstetrical intervention needed) had increased anxiety levels ${ }^{31}$. Conversely, women with anxiety and/or depression during pregnancy were 2.3 times more likely to suffer from arterial hypertension during pregnancy compared to women without anxiety/ or depression ${ }^{30}$; again, demonstrating the interrelationship between maternal physical and mental health during pregnancy.

Women who are obese have been found to be at a higher risk for mental health problems prior to becoming pregnant $^{32}$. One-third of reproductive aged women are obese which has led to an increase in the number of women starting pregnancy as obese (body mass index $\left.(\mathrm{BMI}) \geq 30 \mathrm{~kg} / \mathrm{m}^{2}\right)^{33}$. Obesity itself during pregnancy is associated with both maternal and fetal complications such as preeclampsia, gestational diabetes and the need for a cesarean section ${ }^{34}$. Holton et al., studied 1,621 women and found that $25 \%$ of women who were overweight (25$29.9 \mathrm{~kg} / \mathrm{m}^{2}$ ) or obese prior to pregnancy were anxious compared to women with normal BMI's $\left(<25 \mathrm{~kg} / \mathrm{m}^{2}\right)$ prior to pregnancy ${ }^{35}$. It has also been suggested that obese women are more susceptible to anxiety disorders due to excessive gestational weight gain and/or post-partum weight retention ${ }^{36}$. Cumulatively, these studies indicate the importance of frequent screening of pregnant women for anxiety, but they also suggest that obese women and women with a current high-risk pregnancy or an obstetric complication may benefit from additional psychological support.

\section{Labor and anxiety}

Even if a patienthad smooth antepartum progress, severe anxiety or fear may develop during labor or childbirth ${ }^{37,38}$. Although normal levels of anxiety regarding labor are reported by $6-10 \%$ of pregnant women, many women develop fear or anxiety towards childbirth (tokophobia) ${ }^{37,39}$ . This syndrome is more common in nulliparous women compared to multiparous women ${ }^{40}$. A recent study by Koc et al examined 100 healthy pregnant women and found a direct relationship between the fear of childbirth, anxiety sensitivity and somatosensory amplification in $82 \%$ of the participants ${ }^{41}$. Labor is unpredictable and can occur at any time point, lending to worry about going into labor at an undesirable time (e.g., night, at work, holiday), which may negatively affect access to a desired physician or method of delivery. Some of the other factors that can exacerbate the normal level of anxiety associated with labor include low self-esteem, preexisting medical conditions that have already caused anxiety antepartum, lack of social support and fear of loneliness, fear of pain and a prior negative birth experience ${ }^{38,39}$. Labor anxiety may also stem from the potential complications of vaginal deliveries, such as shoulder dystocia, post-partum hemorrhage, complicated tears, or child morbidities ${ }^{42}$.

Finally, a woman's perception of the healthcare system and her trust with medical staff during her delivery are significant factors that can affect anxiety during delivery. One study found a significant association between a previous negative birth experience (obstetric complications not withstanding) and fear of childbirth among multiparous women ${ }^{43}$. Lack of communication with the patient and/or lack of understanding from the patient can also worsen anxiety levels. This is emphasized in a study by Çankaya and Şimsek who sought to increase patient education by introducing an antenatal education program at week 4 , and as a result, they observed an increase in childbirth self-efficacy, along with the reduction in fear of birth, anxiety, and stress during the pregnancy and post-partum periods ${ }^{44}$. While not all of these sources of anxiety can be eased by education or counseling, prenatal 
education regarding childbirth and maternal complications to help decrease maternal anxiety levels and/or disorders should not be overlooked ${ }^{44,45}$.

\section{Anxiety during the Post-partum period}

During the post-partum period, the number of demands and responsibilities increase, which may also increase worry and distress. Several factors have been found to contribute to the new onset of anxiety and anxiety disorders in women who did not have PrA, OCD or GAD during pregnancy, such as difficulty with breastfeeding, inadequate social support, financial difficulties, maternal health, and child wellbeing ${ }^{46}$. Some studies have reported that anxiety levels decrease during the post-partum period, relative to the prenatal period. A study by Nakic Rados et al., investigated this correlation and found that one in three women who were anxious during pregnancy continued to be anxious in early post-partum while one in two women continued to be anxious in the late post-partum period ${ }^{46}$. However, the most important predictor of progression of anxiety during pregnancy to the 6-week post-partum period was found to be the severity of anxiety experienced during pregnancy. Between $8-12 \%$ of women experience subclinical levels of anxiety during the post-partum period, which is comparable to prevalence rates in the antepartum period ${ }^{47}$. However, this rate is thought to underestimate the prevalence of post-partum anxiety, which is in part, due to problems with the diagnostic criteria ${ }^{48}$. It should be noted, however that OCD tends to increase during the postpartum period, with post-partum exacerbation estimating to range between $25-75 \%{ }^{49}$. As $25-50 \%$ of women with post-partum anxiety or panic disorders will develop postpartum depression, there should be special emphasis on the need for immediate attention and follow up during the post-partum period ${ }^{46}$.

\section{Effects of Maternal Anxiety on Infant Outcome}

Maternal anxiety during pregnancy has its own influences on infant outcomes. The pathophysiology is not well understood but many factors play a role including fetal programing, genetic factors and post-partum environmental factors ${ }^{50}$. The effects of anxiety on maternal behavior can be broad, but it can present as disinterest, disengagement, insensitivity and even harsh parenting. Different types of parenting (Authoritarian, permissive, uninvolved, authoritative) can be seen in anxious mothers ${ }^{51}$. Typically, the authoritarian type leads to both internalizing behavior (anxiety, withdrawal and loneliness) and externalizing behavior (impulsivity, aggressiveness and disruptiveness). Separate from the effects of maternal parenting styles, maternal PrA, peripartum panic disorders and post-partum anxiety disorders are also suggested to influence infant outcome ${ }^{52}$. Parfitt and Ayers reported increased relationship problems after birth for couples that had post-partum anxiety disorders which were associated with women having negative behavior towards/ in response to their significant other and their infants ${ }^{53}$.

Women with PrA are at an increased risk for preterm deliveries, with fetal growth-retardation, low birth weight and/or intrauterine growth restriction ${ }^{54}$. Infants born prematurely, defined by the Centers for Disease Control is birth before 37 weeks of gestation, can suffer from a multitude of complications stemming from several different organ systems. In addition to the developmental problems described above, some of the organ problems affecting premature infants can include respiratory distress syndrome and pneumonia, as well as increased susceptibility to infection and sepsis, patent ductus arteriosus and more commonlynecrotizing enterocolitis ${ }^{5,56}$. The effects of prenatal panic attacks on infant outcomes are not as clear, especially as some studies have reported an association between maternal panic attacks, preterm delivery, the need for cesarean sections and developmental delays in infants ${ }^{57}$. However, other studies have not found evidence to support these negative associations ${ }^{57,58}$.

Prenatal anxiety disorders have been associated with cognitive deficits in the offspring that are independent of any postnatal environmental stress ${ }^{59}$. Maternal anxiety disorders during pregnancy have also been implicated in contributing to socio-emotional problems and temperament difficulties in the offspring, such as high negative affectivity or negative emotionality and poor attentional regulation ${ }^{60}$. A study by Polite et al, assessed women for anxiety disorders before, throughout and up to 2-years post-partum along with their offspring to determine if there was a correlation between the occurrence of anxiety disorders and problems with child development (social-emotional, physical and medical) ${ }^{61}$. They found that children born to women with high levels of anxiety while pregnant had an 0.R. 2.48 (95\% CI 1.55-4.92) of developing social-emotional delays, which increased to O.R. 3.32 among women with elevated postnatal anxiety levels and 0.R. 3.98 in women with both elevated pre and postnatal anxiety levels. Additional studies subsequently described higher rates of behavioral problems and decreased executive working memory in offspring born to women who suffered from prenatal anxiety ${ }^{62,63}$.

Maternal post-partum anxiety affects maternal-infant interaction and potentially the mother's ability to parent and bond with her newborn(s) ${ }^{64}$. Studies report elevated anxiety levels across pregnancy and the post-partum period can lead to poor neonatal feeding and nurturing which is often criticized by the mother ${ }^{65,66}$. Self-recrimination is particularly true among women suffering from post-partum OCD, as studies have found that these women tend to suffer more from feelings of self-blame and guilt. All of which ultimately have an adverse impact on infant bonding ${ }^{67}$. 
Table 1. Common diagnostic measures of anxiety during pregnancy.

\begin{tabular}{|l|c|c|c|}
\hline \multicolumn{1}{|c|}{ Name } & \# Items & Response Format & Description \\
\hline Beck Anxiety Inventory (BAI) & 21 & 4 point Likert Scale & Self-report instrument \\
\hline Cambridge Worry Scale (CWS) & 16 & 6 point Likert Scale & Worry during pregnancy \\
\hline Hospital Anxiety and Depression Scale (HADS-A) & 7 & 4 point Likert Scale & General use and anxious arousal \\
\hline Penn State Worry Questionnaire & 16 & 5 point Likert Scale & General use and assess worry \\
\hline Perinatal Anxiety Screening Scale (PASS) & 31 & 4 point Likert Scale & Perinatal-specific anxieties \\
\hline Pregnancy Anxiety Scale & 5 & Visual Analogue Scale & Assess anxiety in pregnancy \\
\hline Pregnancy Anxiety Scale & 10 & Dichotomous & Multidimensional PrA measurement \\
\hline Prenatal Distress Questionnaire (PDQ) & 12 & 5 point Likert Scale & Pregnancy-specific stress \\
\hline Pregnancy Related Anxiety Questionnaire (PRAQ) & 58 & 7 point Likert Scale & Assess fears and worries in pregnancy \\
\hline Pregnancy Related Thoughts & 10 & 4 point Likert Scale & Assess worry and concern in PrA \\
\hline Pregnancy Specific Anxiety Scale & 4 & 5 point Likert Scale & Assess anxiety in pregnancy \\
\hline State-Trait Anxiety Index (STAI) & 40 & 4 point Likert Scale & State and trait anxiety assessment \\
\hline $\begin{array}{l}\text { Wijma Delivery Expectancy/Experience Questionnaire } \\
\text { (W-DEQ) }\end{array}$ & 33 & 6 point Likert Scale & Assess tokophobia \\
\hline
\end{tabular}

PrA - Pregnancy related Anxiety. An overview of these specific tests are provided in a review by Meades \& Ayers ${ }^{71}$

\section{Assessing and Diagnosing Anxiety During Pregnancy and Post-Partum}

Several self-report questionnaires have been found to be valid and reliable for assessing anxiety during pregnancy and the early post-partum period (Table 1, see Meades \& Ayers for review $\left.{ }^{68}\right)$. These measures vary in the type of anxiety evaluated, and the time for reporting symptoms. Unfortunately, anxiety often goes unidentified, especially if the full DSM-5 criteria are used to determine diagnostic status, as opposed to detecting clinical levels of anxiety based on patient-reported symptomology ${ }^{4}$. Separate from the potential underdiagnoses of anxiety disorders due to the DSM- 5 criteria are the numerous additional barriers to identifying anxiety during pregnancy, including the stigma associated with mental illness, the focus on the physical health of the mother and fetus, or other unidentified factors.

\section{Management of Anxiety in Pregnancy and Post- Partum}

To date, there are several treatment avenues for women affected by anxiety during pregnancy with the primary goal being to prevent the escalation of symptoms while improving the quality of life. Ideally, therapy would begin in the preconception period for women experiencing anxiety or who have an anxiety disorder prior to pregnancy. Non-pharmacologic (psychotherapy) and pharmacologic treatments exist for women who develop an anxiety disorder during pregnancy and the perinatal period. Non-pharmacologic treatments can include psychosocial techniques used to reduce stress (i.e., coping skills, relaxation techniques, diaphragmatic breathing), evidenced-based HVPs and CBT approaches that address maladaptive behaviors and cognition.

\section{Evidence Based HVP}

Evidence-based HVPs are interventions that have been implemented to address maternal mental health in pregnancy and the postpartum period. Some core elements of these intervention programs include strengthen maternal interactions, promoting nurturing behavior, and promoting child well-being ${ }^{69}$. These visits can vary by type and by visitor (nurse, social worker or paraprofessional) and the time spent with each family differs as well; programs can start as early as the prenatal period until the child is at school age ${ }^{69}$. The enrollment strategies in these programs are different and the prevalence of patients enrolled with anxiety during pregnancy or postpartum differs between programs ${ }^{69}$. The effectiveness of HVPs has been somewhat undecided as some studies have reported little to no benefit, due in part to lack of engagement from mothers ${ }^{69,70}$. In an effort to evaluate HVP and their effects on maternal behavior, Nievar et al conducted a meta-analysis which examined 6,453 families $^{71}$. This study reported that HVPs were associated with positive results with there being a direct correlation between the number of visits and maternal outcome. HVPs, again with higher positive outcomes being associated with higher number of visits, were found to be equally effective in improving outcomes among children ${ }^{72}$.

Some discrepancy might also be due to the fact that PrA based interventions are not as well established, with most HVPs focused on holistic behavioral changes instead of dealing with solely anxiety in these pregnant moms. Some of the primary reasons for lack of a noticeable improvement in maternal mental health among HVPs is that home visitors are not well trained to address complex symptoms, moreover the curriculum of the program is not targeted to improve these symptoms. Therefore, implementing change in the curricula of these programs and including mental health providers as a part of the HVP might achieve better outcomes. Even though there exists a lack in focused interventions and somewhat of a conflict in some studies, 
the apparent success of implementing HVPs to mothers provide long term benefits that affects both mother and child $^{73,74}$. The apparent need for focused HVPs are great and continued research and specific programs need to be put in place to better monitor and help women suffering from Pr-A in both the prenatal and postpartum periods.

Used as an umbrella term, CBT refers to short-term, evidence-based interventions that focus on developing skills to change maladaptive cognitive and behavioral patterns to reduce psychological distress. CBT is well-established for the treatment of anxiety-related disorders ${ }^{75}$, and initial work supports its efficacy for improving outcomes for women with anxiety while pregnant ${ }^{76,77}$. Traditional CBT for anxiety disorders incorporates various strategies, such as psychoeducation, cognitive restructuring, problemsolving, exposures, relaxation/coping strategies, and behavioral experiments. These techniques are adapted to address unique concerns and circumstances experienced by women both during pregnancy and the perinatal period. For instance, psychoeducation is modified to incorporate the physiological, social, and psychological adjustments that are common in the perinatal period ${ }^{78}$. In addition, sessions may be compressed into a shorter time period ${ }^{78}$ and scheduled to correspond with regularly scheduled medical appointments or to address critical periods of pregnancy ${ }^{79}$. These interventions have been tested in group $^{78-80}$ and individual formats ${ }^{81}$, with the number of sessions ranging from 2 to 12 sessions. While some studies have evaluated disorder- specific interventions that focus on anxiety pathology ${ }^{78,82}$ or even a specific anxiety disorder, such as blood-injection-injury phobia ${ }^{79}$, other studies have evaluated trans diagnostic interventions for anxiety and depression disorders ${ }^{80,81}$. Despite the small number of high quality randomized controlled trials examining anxiety during pregnancy, a recent meta-analysis found a large effect of CBT for reducing anxiety symptoms from pre- to post-treatment among pregnant women ${ }^{76}$. However, this field of study is limited by the small number of studies evaluating CBT among non-White women. While CBT has not been found to be effective among Black and Latin women $^{83}$, it was affective in alleviating fear in Iranian women $^{84}$. Nevertheless, continued research is needed to further examine the efficacy and effectiveness of CBT for anxiety during pregnancy and even in the post-partum period. In particular, the clinical care and outcomes of this vulnerable population may be further improved through future studies that emphasize the recruitment of diverse samples, continued efforts to increase access to care through considerations of affordability and feasibility, and comprehensive evaluation of short- and long-term maternal and infant outcomes.

Some women are treated via a pharmacologic intervention when non-pharmacologic treatment is believed to have no benefit ${ }^{85}$. Two categories of medications are commonly considered, antidepressants and benzodiazepines. Selective Serotonin reuptake inhibitors (SSRIs) have been shown to improve some forms of anxiety during pregnancy ${ }^{86}$. Depending on the dose and drug taken, SSRIs are relatively effective for the mother; however there have been reports of little to no increases in major congenital malformations or neurodevelopmental and behavioral effects in offspring ${ }^{86}$. Additionally, some women experience hypertension during pregnancy and a shorter gestation when taking SSRIs during pregnancy ${ }^{57}$. Benzodiazepines, if taken during the $1^{\text {st }}$ trimester have been associated with teratogenic effect in the fetus, whereas if they are taken in the $3^{\text {rd }}$ trimester neonatal withdrawal system has been reported ${ }^{57}$. Infants who were exposed to benzodiazepines in utero have an increased need for ventilator support ${ }^{57}$. Avoiding these medications in pregnancy or at least tapering them in the third trimester if they are needed is recommended. Despite the number of women, using pharmacologic therapy to address anxiety disorders, the long-term effects of prenatal pharmacotherapy use on the developing fetus are unclear.

\section{Conclusion}

Elevated anxiety levels and the onset of anxiety disorders during pregnancy and in the post-partum period has been increasing and is highly prevalent ${ }^{87}$. Given the large degree of overlap and the frequent co-occurrence of depressive and anxiety disorders during pregnancy and in the post-partum period it is not always easy to differentiate these two disorders; continued research is needed to further parse the differences and similarities between them. Further complicating the diagnosis of anxiety is the timing of the interview or survey. According to the DSM5, anxiety disorders overlap with one another as they can share excessive fear and behavioral disturbances ${ }^{7}$. However, anxiety disorders differ from one another through the type of situation. In the case of PrA and postpartum anxiety, one diagnostic problem with the DSM-5 criteria for anxiety is the mandate for excessive worries that occur for at least 6 months ${ }^{88}$. Based on this diagnosis alone some pregnant/post-partum women may potentially be excluded. Therefore, some researchers define GAD during pregnancy if the illness meets other DSM criteria for a minimum duration of one month ${ }^{89}$.

Screening and education are necessary components of prenatal care with the implementation of multidisciplinary approaches to tackle the care of maternal anxiety. Early counseling and screening are needed to achieve good outcomes during pregnancy. Additionally, screening should not be limited to one time during the antepartum period, but at least once per trimester as well as immediately after childbirth to assess maternal status. Screening has mainly focused on depression, which leads to missing 
anxiety-related diagnosis that present without depressive symptoms. Anxiety and post-partum depression can overlap and cause a comorbid condition; however, screening should aim to differentiate these two conditions in order to have a targeted screening approach. Further supporting non-pharmacological approaches, studies have shown health risks associated with pharmacotherapy ${ }^{85}$ and shown women are reluctant to use pharmacotherapy due to side effects and risks associated with fetal exposure ${ }^{90}$. Specifically, compared to pharmacotherapy, Arch and colleagues found pregnant women reported a higher willingness to engage in CBT and fewer treatmentrelated concerns for $\mathrm{CBT}^{90}$. However, more studies need to be conducted to ensure that CBT and other nonpharmacological avenues are effective for all populations and pharmacologic interventions are thoroughly vetted for safety use during pregnancy for the mother and infant.

Though this review is an overview of the selected literature and does not systematically review every study on maternal anxiety that has been published, there have been several systematic reviews published over the past few years that have specifically examined the role of different factors on maternal anxiety (i.e., microbiome ${ }^{91}$, paternal input ${ }^{92}$, pharmacological vs non-pharmacological interventions ${ }^{93}$ ). The role of the immune system in contributing to anxiety and depression has been extensively covered and if appropriate biomarkers were developed, this could be one potential way in which anxiety and depression may be able to be distinguished. Additionally, during pregnancy the unique immunological state requires a balance of immune tolerance and suppression in order to protect the fetus without compromising the mother ${ }^{94}$. Imbalances in the immune system ${ }^{95}$, hormones ${ }^{96}$ and the hypothalamic pituitary adrenal $\left(\mathrm{HPA}^{97}\right)$ axis have all been suspected of contributing to anxiety levels both during pregnancy and outside of pregnancy. However, despite the work performed in these areas, the evidence to confirm a definitive mechanism or the utility of these systems for therapeutic targeting or diagnosis is still lacking ${ }^{98,99}$. Future studies should also consider the impact of anxiety and strive to consider if health care workers should be doing more for women in the perinatal period then routinely administering surveys during the post-partum period. Additional studies examining anxiety itself separate from depression are needed to determine the full impact of increased anxiety on maternal health, post-partum recovery and bonding with the infant.

The current review summarizes what is currently known regarding anxiety during pregnancy and the postpartum period by integrating biological, environmental and psychological information. We can conclude from both our review and that of others that the mechanisms leading from maternal anxiety to infant outcome still need better understanding to implement specific interventions. Despite the increased awareness of maternal mood, further work is needed to ensure prenatal mental health is monitored as closely as physical health in light of their reciprocal relationship. Furthermore, beyond the accurate identification of high anxiety risk or the presence of pathological anxiety, effective interventions for mother and infant are critical to reduce anxiety and improve mental health outcomes.

\section{Acknowledgments}

Research reported in this publication was supported by the National Institute of General Medical Sciences of the National Institutes of Health under Award Number R01MH116027 to KW and T32HL105324 to AG. The content is solely the responsibility of the authors and does not necessarily represent the official views of the National Institutes of Health.

\section{References}

1. Dhillon A, Sparkes E, Duarte RV, et al. Mindfulness-based interventions during pregnancy: A systematic review and meta-analysis. Mindfulness. 2017; 8(6): 1421-1437.

2. Verbeek T, Arjadi R, Vendrik JJ, et al. Anxiety and depression during pregnancy in Central America: A cross-sectional study among pregnant women in developing country Nicaragua. BMC Psychiatry. 2015; 15: 292.

3. Committee A. Clinical management guidelines for obstetriciangynecologists: use of psychiatric medications during pregnancy and lactation. ACOG Practice Bulletin No. 92. Obstet and Gynecology. 2008; 111(4): 1001-1020.

4. Furtado M, Chow CHT, Owais S, et al. Risk factors of new onset anxiety and anxiety exacerbation in the perinatal period: A systematic review and meta-analysis. J Affect Disord. 2018; 238: 626-635.

5. Wenzel A. Anxiety in childbearing women: Diagnosis and treatment. 2011.

6. Field T, Diego M, Hernandez-Reif M, et al. Comorbid depression and anxiety effects on pregnancy and neonatal outcome. Infant Behav Dev. 2010; 33(1): 23-29.

7. Association AP. Diagnostic and Statistical Manual of Mental Disorders (DSM-5). 5 Arlington, VA: American Psychiatric Publishing. 2013.

8. Ali E. Women's experiences with postpartum anxiety disorders: A narrative literature review. Int J Women's Health. 2018; 10: 237-249.

9. Watterson RA, Williams JV, Lavorato DH, et al.Descriptive epidemiology of generalized anxiety disorder in Canada. The Canadian Journal of Psychiatry. 2017; 62(1): 224-229.

10. Phillips J, Sharpe L, Matthey S, et al. Rates of depressive and anxiety disorders in a residential mother-infant unit for unsettled infants. Australian and New Zealand Journal of Psychiatry. 2007; 41: 836-842.

11. Grigoriadis S, Meschino DDC, Barrons E, et al. Mood and anxiety disorders in a sample of Canadian perinatal women referred for psychiatric care. Arch Womens Ment Health. 2011; 14(4): 325-333.

12. Huizink AC, Mulder EJ, Robles de Medina PG, et al. Is pregnancy anxiety a distinctive syndrome? Early Hum Dev. 2004; 79: 81-91.

13. Bayrampour H, Ali E, McNeil DA, et al. Pregnancy-related anxiety: A concept analysis. Int J Nurs Study. 2016; 55: 115-130.

14. Mudra S, Göbel A, Barkmann C, et al. The longitudinal course of 
pregnancy-related anxiety in parous and nulliparous women and its association with symptoms of social and generalized anxiety. J Affect Disord. 2020; 260: 111-118.

15. Russell E, Fawcett JM, Mazmanian D, et al. Risk of obsessivecompulsive disorder in pregnant and postpartum women: A metaanalysis. J Clin Psychiatry. 2013; 74: 377-385.

16. Burton H. How women with established obsessive compulsive disorder experience pregnancy and postpartum: an interpretative phenomenological analysis. J Reprod Infant Psychol. 2020; 30: 1-13.

17. Viswasam K, Eslick GD, Starcevic V, et al. Prevalence, onset and course of anxiety disorders during pregnancy: A systematic review and meta analysis. J Affect Disord. 2019; 255: 27-40.

18. Harpel T. Fear of the unknown: ultrasound and anxiety about fetal health. Health. 2008; 3: 295-312.

19. van de Loo KFE, Vlenterie R, Nikkels SJ, et al. Depression and anxiety during pregnancy: The influence of maternal characteristics. Birth 2018; 45(4): 478-489.

20. Feligreras-Alcalá D, Frías-Osuna A, Del-Pino-Casado R, et al. Persona and Family Resources Related to Depressive and Anxiety Symptoms in women during Puerperium. Int J Environ Res Public Health. 2020; 17(14): 5230.

21. Yu M, Qiu T, Liu C, et al. The mediating role of perceived social support between anxiety symptoms and life satisifcation in pregnant women: A cross-sectional study. Health Qual Life Outcomes. 2020; 18(1): 223.

22. Orr S. Social support and pregnancy outcome: A review of the literature. Clin Obstet Gynecol. 2004; 47(4): 842-855.

23. Kim M, Lee SM, Bae SH, et al. Socioeconomic status can affect pregnancy outcomes and complications, even with a universal healthcare system. Int J Equity Health. 2018; 17(1): 2.

24. Martini J, Asselmann E, Einsle F, et al. A prospective-longitudinal study on the association of anxiety disorders prior to pregnancy and pregnancy-and child-related fears. J Anxiety Disord. 2016; 40: 58-66.

25. Coco L, Giannone TT, Zarbo G. Management of High-Risk Pregnancy. Minerva Ginecol. 2014; 66(4): 383-389.

26. Abrar A, Shepherd E, Middleton P, et al. Anxiety among women experiencing medically complicated pregnacy: A systematic review and meta-analysis. Birth. 2020; 47(1): 13-20.

27. Fairbrother $\mathrm{N}$, Young $\mathrm{AH}$, Zhang $\mathrm{A}$, et al. The prevalence and incidence of perinatal anxiety disorders among women experiencing a medically complicated pregnancy. Arch Womens Ment Health. 2017; 20(2): 311319 .

28. Arulkumaran S. Diabetes in pregnancy. Best Pract Res Clin Obstet Gynaecol. 2011; 25(1): 1.

29. ACOG. Hypertension in pregnancy. Report of the ACOG Task force on Hypertension in Pregnancy. Obstet Gynecol. 2013; 122(5): 1122 1131.

30. Winkel S, Einsle F, Pieper L, et al. Associations of anxiety disorders, depressive disorders and body weight with hypertension during pregnancy. Arch Womens Ment Health. 2015; 18(3): 473-483.

31. Fischbein RL, Nicholas L, Kingsbury DM, et al. State Anxiety in Pregnancies Affected by Obstetric Complications: A systematic review. J Affect Disord. 2019; 257: 214-240.

32. Keitt S, Resnick EM, Simon VR, et al. Behavior and obesity in women across the life span: A report by the Society for Women's Health Research. J Investig Med. 2008; 56(6): 830-842.

33. Flegal KM, Carroll MD, Kit BK, et al. Prevalence of obesity and trends in the distribution of body mass index among US adults, 1999-2010. JAMA. 2012; 307(5): 491-497.

34. Ovesen P, Rasmussen S, Kesmodel U. Effect prepregnancy maternal overweight and obesity on pregnancy outcome. Obstet and Gynecology. 2011; 118: 305-312.

35. Holton S, Fisher J, Nguyen H, et al. Pre-pregnancy body mass index and the risk of antenatal depression and anxiety. Women and Birth. 2019; 32(6): e508-e514.

36. Nagl M, Linde $\mathrm{K}$, Stepan $\mathrm{H}$, et al. Obesity and anxiety during pregnancy and postpartum: A systematic review. J Affect Disord. 2015; 186: 293305.

37. Richens Y, Smith DM, Lavender DT. Fear of birth in clinical practice: A structured review of current measurement tools. Sexual \& Reproductive Healthcare. 2018; 16: 98-112.

38. Dencker A, Nilsson C, Begley C,. Causes and outcomes in studies of fear of childbirth: A systematic review. Women Birth. 2019; 32(2): 99-111.

39. Hofberg K, Ward M. Fear of pregnancy and childbirth. Postgrad Med J. 2003; 79: 505-510.

40. Khwepeya M, Lee GT, Chen SR, et al. Childbirth fear and related factors among pregnant and postpartum women in Malawi. BMC Pregnancy and Childbirth. 2018; 18: 391.

41. Koc A, Colak S, Colak GV, et al. Investigating fear of childbirth in pregnant women and its relationship between anxiety sensitivity and somatosensory amplification. Journal of Obstetrics and Gynaecology. 2020 .

42. Johnson R, Slade P. Obstetric complications and anxiety during pregnancy: Is there a relationship? J Psychosom Obstet Gynecol. 2003; 24(1): 1-14.

43. Størksen HT, Garthus-Niegel S, Vangen S, et al. The impact of previous birth experiences on maternal fear of childbirth. Acta Obstet Gynecol Scand. 2013; 92(3): 318-324.

44. Cankaya S, Simsek B. Effects of antenatal education on fear of birth, depression, anxiety, childbirth self-efficacy, and mode of delivery in primiparous pregnant women: A prospective randomized controlled study. Clin Nurs Res. 2020.

45. Fenwick J, Toohill J, Slavin V, et al. Improving psychoeducation for women fearful of childbirth: Evaluation of a research translation project. Women and Birth. 2018; 31: 1-9.

46. Nakić Radoš S, Tadinac M, Herman R. Anxiety During Pregnancy and Postpartum: Course, Predictors and Comorbidity with Postpartum Depression. Acta Clin Croat. 2018; 57(1): 39-51.

47. Goodman JH, Chenausky KL, Freeman MP. Anxiety disorders during pregnancy: A systematic review. J Clin Psychiatry. 2014; 75: e1153-e1184.

48. Fairbrother N, Janssen P, Antony MM,. Perinatal anxiety disorder prevalence and incidence. Journal of Affective Disorders. 2016; 200: 148-155.

49. Uguz F, Ayhan M. Epidemiology and clinical features of obsessivecompulsive disorder pregnancy and postpartum period: a review. J Mood Disord. 2011; 1: 178-186.

50. Dowse E, Chan S, Ebert L. Impact of perinatal depression and anxiety on birth outcomes: A retrospective data analysis. Maternal and Child Health Journal. 2020; 24(6): 718-726.

51. Huizink AC, Menting B, De Moor MHM, et al. From prenatal anxiety to parenting stress: A longitudinal study. Arch Womens Ment Health. 2017; 20(5): 663-672.

52. Martini J, Beesdo-Baum K, Garthus-Niegel S, et al. The course of panic disorder during the peripartum period and the risk for adverse child development: A prospective-longitudingal study. J Affect Disord. 2020; 266: 722-730.

53. Parfitt Y, Ayers S. Transition to parenthood and mental health in firsttime parents. Infant Mental Health Journal. 2014; 35(3): 263-273. 
54. Hedegaard M, Henriksen TB, Sabroe S, et al. Psychological distress in pregnancy and preterm delivery. Br Med J 1993; 307: 234-239.

55. Goldenberg RL, Culhane JF, Iams JD, et al. Epidemiology and causes of preterm birth. Lancet. 2008; 371(9606): 75-84.

56. Yaari M, Treyvaud K, Lee KJ, et al. Preterm birth and maternal mental health: Longitudinal trajectors and predictors. J Pediatr Psychol. 2019; 44(6): 736-747.

57. Bánhidy F, Acs N, Puhó E, et al. Association between maternal panic disorders and pregnancy complicatiosn and delivery outcomes. Eur J Obstet and Gynecol Reprod Biol. 2006; 124: 47-52.

58. Yonkers K, Gilstad-Hayden K, Forray A, et al. Association of Panic Disorder, Generalized Anxiety Disorder, and Benzodiazepine Treatment During Pregnancy with Risk of Adverse Birth Outcomes. JAMA Psychiatry. 2017; 74(11): 1145-1152.

59. Lin Y, Xu J, Huang J, et al. Effects of prenatal and postnatal maternal emotional stress on toddlers' cognitive and temperamental development. J Affect Disord. 2017; 207: 9-17.

60. Baibazarova E, Beek CVD, Cohen-Kettenis PT, et al. Influence of prenatal maternal stress, maternal plasma cortisol and cortisol in the amniotic fluid on birth outcomes and child temperament at 3 months. Psychoneuroendocrinology. 2013; 38(6): 907-915.

61. Polite C, Junge C, Soest TV, et al. Impact of maternal perinatal anxiety on social-emotional development of 2-year olds, a prospective study of Norwegian mothers and their offspring: The Impact of Perinatal Anxiety on Child Development. Maternal and Child Health Journal. 2019; 23: 386-396.

62. Loomans E, Stelt OVD, Eijsden MV, et al. Antenatal maternal anxiety is associated with problem behaviour at age five. Early Hum Dev. 2011; 87(8): 565-570.

63. Pearson R, Bornstein MH, Cordero M, et al. Maternal perinatal mental health and offspring academic achievement at age 16: the mediating role of childhood executive function. J Child Psychol Psychiatry. 2016; 57(4): 491-501.

64. Martini J, Petzoldt J, Einsle F, et al. Risk factors and course patterns of anxiety and depressive disorders during pregnancy and after delivery: A prospective-longitudinal study. J Affect Disord. 2015; 175: 385-395.

65. Fallon V, Halford JCG, Bennett KM, et al. Postpartum-specific Anxiety as a Predictor of Infant-feeding Outcomes and Perceptions of InfantFeeding behaviours: New Evidence for Childbearing Specific Measures of Mood. Arch Womens Ment Health. 2018; 21: 181-191.

66. Drake K, Ginsburg G. Parenting Practices of Anxious and Non-anxious mothers: A multi-method multi-informant approach. Child Fam Behav Ther. 2011; 33(4): 299-321.

67. Starcevic V, Eslick GD, Viswasam K, et al. Symptoms of ObsessiveCompulsive Disorder during pregnancy and the postpartum period A Systematic review and meta-analysis. Psychiatric Quarterly. 2020.

68. Meades R, Ayers S. Anxiety measures validated in perinatal populations: a systematic review. J Affect Disord. 2011; 133(1-2): $1-15$.

69. Ammerman RT, Putnam FW, Bosse NR, et al. Maternal Depression in Home Visitation: A Systematic Review. Aggress Violent Behav. 2010; 15(3): 191-200.

70. Stevens J. Depression and trauma history in first-time mothers receiving home visitation. Journal of Community Psychology. 2002; 30(5): 551-564.

71. Nievar MA, Van Egeren LA, Pollard S. A meta-analysis of home visiting programs: Moderators of improvements in maternal behavior. Infant Ment Health J. 2010; 31(5): 499-520.

72. Peacock S, Konrad S, Watson E, et al. Effectiveness of home visiting programs on child outcomes: a systematic review. BMC Public Health. 2013; $13: 17$

73. Slade P, Balling K, Sheen K, et al. Establishing a valid construct of fear of childbirth: findings from in-depth interviews with women and midwives. BMC Pregnancy Childbirth. 2019; 19(1): 96.

74. Korfmacher J, Frese M, Gowani S. Examining program quality in early childhood home visiting: From infrastructure to relationships. Infant Ment Health J. 2019; 40(3): 380-394.

75. Carpenter JK, Andrews LA, Witcraft SM, et al. Cognitive behavioral therapy for anxiety and related disorders: A meta-analysis of randomized placebo-controlled trials. Depress Anxiety. 2018; 35(6): 502-514.

76. Maguire PN, Clark GI, Wootton BM. The efficacy of cognitive behavior therapy for the treatment of perinatal anxiety symptoms: A preliminary meta-analysis. J Anxiety Disord. 2018; 60: 26-34.

77. Loughnan SA, Wallace M, Joubert AE, et al. A systematic review of psychological treatments for clinical anxiety during the perinatal period. Arch Womens Ment Health. 2018; 21(5): 481-490.

78. Green SM, Haber E, Frey BN, et al. Cognitive-behavioral group treatment for perinatal anxiety: a pilot study. Arch Womens Ment Health. 2015; 18(4): 631-638.

79. Lilliecreutz C, Josefsson A, Sydsjö G, et al. An open trial with cognitive behavioral therapy for blood- and injection phobia in pregnant women-a group intervention program. Arch Womens Ment Health. 2010; 13(3): 259-265.

80. Bittner A, Peukert J, Zimmermann C, et al. Early intervention in pregnant women with elevated anxiety and depressive symptoms: efficacy of a cognitive-behavioral group program. J Perinat Neonatal Nurs. 2014; 28(3): 185-195.

81. Milgrom J, Holt C, Holt CJ, et al. Feasibility study and pilot randomised trial of an antenatal depression treatment with infant follow-up. Archives of women's mental health. 2015; 18(5): 717-730.

82. Luberto CM, Park ER, Goodman JH. Postpartum Outcomes and Formal Mindfulness Practice in Mindfulness-Based Cognitive Therapy for Perinatal Women. Mindfulness (N Y). 2018; 9(3): 850-859.

83. Ponting C, Mahrer NE, Zelcer H, et al. Psychological interventions for depression and anxiety in pregnant Latina and Black womn in the United States: A systematic review. Clin Psychol Psychother. 2020; 27(2): 249-265.

84. Shahsavan F, Akbari N, Gharraee B, et al. The effect of internet-based guided self-help cognitive-behavioral therapies on Iranian women's psychological symptoms and preferred method of childbirth. Perspect Psychiatr Care. 2020.

85. Marchesi C, Ossola P, Amerio A, et al. Clinical management of perinatal anxiety disorders: A systematic review. J Affect Disord. 2016; 190: 543-550.

86. Singal D, Chateau D, Struck S, et al. In utero antidepressants and neurodevelopmental outcomes in kindergarteners. Pediatrics. 2020; 145(5): e20191157.

87. McKee K, Admon LK Winkelman TNA, et al. Perinatal mood and anxiety disorders, serious metnal illness, and delivery-related health outcomes, United Staes, 2006-2015. BMC Women's Health. 2020; 20(1): 150

88. Kupfer D. Anxiety and DSM-5. Dialogues Clin Neurosci. 2015; 17(3): 245-246.

89. Buist A, Gotman N, Yonkers KA. Generalized anxiety disorder: Course and risk factors in pregnancy. J Affect Disord. 2011; 131: 277-283.

90. Arch JJ. Cognitive behavioral therapy and pharmacotherapy for anxiety: treatment preferences and credibility among pregnant and non-pregnant women. Behav Res Ther. 2014; 52: 53-60. 
91. Redpath N, Rackers HS, Kimmel MC, et al. The relationship between perinatal mental health and stress: A review of the microbiome. Curr Psychiatry Rep. 2019; 21(3): 18.

92. Schmied V, Johnson M, Naidoo N, et al. Maternal mental health in Australia and New Zealand: A review of longitudinal studies. Women and Birth. 2013; 26(3): 167-178.

93. Thorsness K, Watson C, LaRusso EM, et al. Perinatal anxiety: Approach to diagnosis and management in teh obstetric setting. Am J Obstet Gynecol. 2018; 219(4): 326-345.

94. Erlebacher A. Immunology of the maternal-fetal interface. Annu Rev Immunol. 2013; 31: 387-411.

95. Osborne L, Brar A, Klein SL, et al. The role of Th17 cells in the pathophysiology of pregnancy and perinatal mood and anxiety disorders. Brain Behav Immun. 2019; 76: 7-16.
96. Altemus M. Neuroendocrine Networks and Functionality. Med Clin North Am. 2019; 103(4): 601-612.

97. Glynn L, Davis EP, Sandman CA. New insights into the role of perinatal HPA-axis dysregulation in postpartum depression. Neuropeptides. 2013; 47: 363-370.

98. Fassaie S, McAloon J. Maternal distress, HPA activity, and antenatal interventions: A Systematic Review. Psychoneuroendocrinology. 2020; 112: 104477.

99. Leff-Gelman P, Mancilla-Herrera I, Flores-Ramos M, et al. The immune system and the role of inflammation in perinatal depression. Neurosci Bull. 2016; 32(4): 398-420. 